\title{
LIPOPROTÉINES ET MALARIA :
}

\section{Rôle immunorégulateur des complexes immunoglobulines- lipoprotéines de souris infectées par Plasmodium chabaudi sur des cellules productrices d'anticorps}

\author{
D. CAMUS, P. GOUMARD, A. VERNES
}

RÉSUMÉ. Le rôle et le mécanisme d'action des lipoprotéines provenant de souris infectées par Plasmodium chabaudi, sur des cellules sécrétantes d'anticorps ont été recherchés. Des lipoprotéines ou des complexes Ig-lipoprotéines sont introduits dans des cultures de cellules spléniques provenant de souris préalablement immunisées vis-à-vis de l'anatoxine tétanique. Les taux d'anticorps antitétaniques sécrétés dans les surnageants de ces cultures sont déterminés par dosage radio-immunométrique utilisant une anatoxine purifiée, marquée à l'iode-125 et un sérum antitétanique de référence titré en unités internationales. Les lipoprotéines provenant de sérums de souris normales ou infectées depuis $5,7,21$ ou 28 jours par $P$. chabaudi n'inhibent pas l'activité des cellules sécrétrices d'anticorps antitétaniques. A l'inverse, les lipoprotéines provenant de souris infectées depuis 11 ou 13 jours (naturellement complexées à des immunoglobulines) inhibent la production in vitro d'anticorps antitétaniques. Les complexes formés par incubation de Lp J-7 avec des sérums provenant de souris infectées depuis 20 jours ou la fraction IgG de ces sérums, inhibent les cellules sécrétrices d'anticorps. Toutefois, lorsque ces cellules sont pré-incubées avec des fragments Fc d'IgG, l'activité inhibitrice des complexes Ig-Lp isolés des sérums de souris infectées depuis 13 jours et des complexes formés in vitro n'est plus observée. Ces résultats suggèrent que les lipoprotéines de souris infectées par $P$. chabaudi nécessitent d'être sous forme d'immun-complexes pour exercer un rôle inhibiteur sur des cellules sécrétrices d'anticorps. La présence des complexes Ig-Lp en cours d'infection par $P$. chabaudi pourrait expliquer le rôle immunodépresseur de l'infection malarique sur une réponse humorale, à des antigènes hétérologues, pré-existante à l'infection parasitaire.

\section{Lipoproteins and Malaria : II. Immunoregulation by immunoglobulin-lipo- protein complexes from Plasmodium chabaudi infected mice on antibody secreting cells.}

SUMMARY. Injection of lipoproteins from Plasmodium chabaudi infected mice into mice previously immunized with either human serum transferrin, bovine serum albumin, polyvinylpyrrolidone or tetanus toxoid, was followed by a decrease in the levels of antibodies directed

INSERM U 42, Biologie et Biochimie parasitaires et fongiques, 369, rue Jules-Guesde, F 59650, Villeneuve d'Ascq, Laboratoire de Parasitologie et Mycologie, Faculé de Médecine, Lille.

Accepté le 2 novembre I 984 .

Abréviations utilisées dans le texte : $\operatorname{Ig} G-L p$ : complexes immunoglobulines G-lipoprotéines; $L D L$ : lipoprotéines de basse densité; $L p N$ : lipoprotéines isolées de sérums de souris non infectées ; $L p J-n$ : lipoprotéines isolées de sérums de souris infectées par $P$. chabaudi depuis $n$ jours ; ser $J-x$ : sérum provenant d'une souris infectée par $P$. chabaudi depuis $x$ jours ; $(L p J-n)-(\operatorname{ser} J-x)$ : complexe formé par incubation de $\mathrm{Lp}$ J-n et de ser J-x. 
against these antigens, suggesting a blockade of antibody secreting cells (Goumard et al., 1982). However, lipoproteins in $P$. chabaudi infected mice are complexed with immunoglobulins during the second week of infection (Demonchy et al., 1982). In this study, the effects of lipoproteins and Ig-lipoprotein complexes (Ig-Lp) on antibody secreting cells was investigated in vitro. Spleen cells from mice immunized with tetanus toxoid were cultured in microplates and the antitetanus antibodies (anti-TT Ab) were measured in the culture supernatants using a radioimmunoassay (Goumard et al., 1984). Ig-Lp purified from day-11 or day-13 $P$. chabaudi infected mice inhibited the secretion of anti-TT $\mathrm{Ab}$ when introduced into microcultures. On the contrary, lipoproteins purified from either day-5, day-7, day-21 or day-28 infected mice as well as lipoproteins from uninfected mice did not inhibit anti-TT $\Lambda$ b secreting cells. Ig-L $\mathrm{p}$ formed in vilro with lipoproteins purified from day 7 infected mice $\left(\mathrm{L}_{\mathrm{p}} \mathrm{J}-7\right)$ and day-20 infected mice sera, inhibited anti-TT $\mathrm{Ab}$ secreting cells. IgG purified from day-20 sera and incubated with $\mathrm{Lp} \mathrm{J-7}$, inhibited anti-TT Ab secreting cells but no inhibitory effect was observed with the $\mathrm{F}\left(\mathrm{ab}^{\prime}\right) 2$ fragments of these Jg. Pre-incubation of anti-TT Ab secreting cells with $\mathrm{Fc}$ fragments of mouse IgG blocked the inhibitory eflect of Ig-Lp purified from infected mouse sera or formed in vitro. Our results indicate that lipoproteins from $P$. chabaudi infected mice need to be complexed to exert their inhibitory eflect on antibody secreting cells. The occurence of Ig- $L$ p during malarial infection could explain the influence of the parasitic infection on the antibody response to heterologous antigens previously described (Goumard et al., 1982).

\section{Introduction}

Les infections parasitaires, naturelles ou expérimentales, déterminent chez l'hôte des troubles importants des mécanismes d'immunorégulation (Capron et coll., 1977). Nous avons pour notre part observé que l'infection de la souris Swiss par Plasmodium chabaudi altère les réponses anticorps à des antigènes hétérologues, pré-existantes à l'infection parasitaire. En effet, chez des souris préalablement immunisées contre divers antigènes (transferrine sérique humaine, sérum albumine bovine, anatoxine tétanique, polyvinylpyrrolidone), l'infection palustre provoque une baisse de la capacité de fixation des sérums et du taux des anticorps dirigés contre ces antigènes. Nous avons de plus déterminé que l'injection de lipoprotéines de basse densité provenant de souris infectées depuis 9 jours, à des souris immunisées contre l'anatoxine tétanique, diminue significativement la production d'anticorps antitétaniques (Goumard et coll., 1982). Ces résultats suggéraient donc que les lipoprotéines qui apparaissent en cours de l'infection palustre peuvent altérer la production d'anticorps.

Nous avons ultérieurement rapporté que l'infection de la souris Swiss par $P$. chabaudi s'accompagne de l'apparition, dans le sérum, de complexes immunoglobulines-lipoprotéines. Ceux-ci sont détectés 9 jours après le début de l'infection, atteignent un taux maximum au treizième jour et disparaissent entre le seizième et le vingt et unième jour après le début de l'infection (Demonchy et coll., 1982).

Le but du présent travail est donc de déterminer si l'action immunorégulatrice des lipoprotéines de souris infectées par $P$. chabaudi s'exerce sur des cellules sécrétantes d'anticorps et nécessite un complexe immunoglobuline-lipoprotéine. 


\section{Matériels et méthodes}

\section{Infection par Plasmodium chabaudi, Dosage des complexes Immunoglobulines- Lipoprotéines (Ig-Lp), Dosage des anticorps anti-lipoprotéines.}

Ces différentes investigations ont été menées conformément aux protocoles précédemment définis (Camus et coll., 1985).

Isolement des lipoprotéines.

En raison de la formation de complexes Ig-Lp en cours d'infection, la purification des lipoprotéines ne peut être réalisée avec fiabilité par collecte de fractions sériques de différentes densités (Gilvery et Goldstein, 1983). Il a donc été préféré une technique de purification basée sur une chromatographie d'affinité. Les sérums de souris normales ou infectées sont fractionnés sur colonne d'héparine Ultrogel A4R (IBF, Villeneuve-la-Garenne, France). Le tampon de départ est un tampon borate $\mathrm{pH} 8.4$ contenant $1 \mathrm{mM}$ de $\mathrm{Ca}++$ et $1 \mathrm{mM}$ de $\mathrm{Mg}++$. L'élution est réalisée avec une solution d'héparine à 500 unités/ $\mathrm{ml}$ dans le tampon de départ selon les recommandations de Pan et coll. (1978). L'éluat est filtré sur membrane Amicon X100 et reconcentré de façon à obtenir 5 unités-lipoprotéines (U-Lp)/ml. L'U-Lp a été déterminée selon le protocole précédemment défini (Camus et coll., 1985). Pour chaque fraction collectée, la présence d'apoprotéine B est contrôlée par un Rocket Apofilm Assay (Sebia, Issy-les-Moulineaux, France) et la présence d'IgG par immunonéphélométrie.

Production in vitro et dosage des anticorps antitétaniques.

La technique précédemment décrite par Goumard et coll. (1984) a été utilisée au cours du présent travail. En résumé, les cellules spléniques proviennent de souris Swiss immunisées vis-à-vis de l'anatoxine tétanique (Institut Pasteur, Paris) recevant une injection de rappel 16 heures avant la mise en culture des cellules. Les cellules sont cultivées en microplaques à fond plat, à raison de $10^{6}$ lymphocytes/ 200 microlitres en présence de LPS (50 microgrammes) et de PWM (50 ui) (Difco, Courbevoie, France). Les fractions à tester sont introduites sous un volume de 20 microlitres. Après 7 jours de culture, les surnageants sont récupérés et le dosage des anticorps antitétaniques est réalisé par une incubation avec de l'anatoxine tétanique $5 \times 10^{-2} \mathrm{ULF} /$ tube couplée au CNBr-Sépharose (Pharmacia, Parly 2, France) suivie de l'addition d'anatoxine marquée à l'iode-125. Après lavage, la radioactivité du culot est mesurée dans un compteur Minigamma (LKB, Orsay, France). Les taux d'anticorps sont exprimés en unités internationales à partir d'une courbe étalon établie grâce à un immun sérum standard titré (Institut Pasteur, Paris). Cette méthode permet de détecter des taux d'anticorps de $0,05 \mathrm{ui} / \mathrm{ml}$ avec des variations intra et inter-séries de 4 et $6 \%$ respectivement. 
Isolement des IgG et purification des fragments $F\left(a b^{\prime}\right) 2$ et des fragments $F c$ des $I g G$.

L'isolement des IgG a été effectué par absorption des sérums sur protéine A-sépharose selon les recommandations du fabricant (Pharmacia, Parly 2, France). Les IgG sont éluées par un tampon citrate $0,1 \mathrm{M}$ à $\mathrm{pH} \mathrm{3,5.} \mathrm{Le} \mathrm{pH}$ des fractions éluées est ajusté à 7,5-8 avec un tampon Na2-C03 l M. Après contrôle en immunoélectrophorèse avec un sérum de lapin anti-IgG de souris, les fractions contenant les IgG sont lyophilisées.

La purification des fragments $F\left(a b^{\prime}\right) 2$, après protéolyse des $\operatorname{Ig} G$ à la pepsine, a été effectuée selon le protocole de Stanworth et Turner (1978) par gel filtration successives sur Séphadex G-150 et G-200. L'isolement des fragments Fc a été réalisé par digestion à la papaine d'IgG de souris (Miles, Paris) et filtration sur colonne de DEAE-cellulose (Hudson et Hay, 1980). Les fractions contenant les fragments $\mathrm{F}\left(\mathrm{ab}^{\prime}\right) 2$ et $\mathrm{Fc}$ sont lyophilisées après contrôle en immunoélectrophorèse avec un sérum de lapin anti-IgG de souris.

Formation de complexes Immunoglobulines-Lipoprotéines in vitro.

Sérums, IgG purifiées ou fractions $F\left(\mathrm{ab}^{\prime}\right) 2$ des IgG sont incubés 1 heure à $37^{\circ} \mathrm{C}$ avec différentes fractions lipoprotéiniques dans les proportions suivantes : a-pour un sérum non fractionné, $0,25 \mathrm{ml} / 1 \mathrm{U}-\mathrm{Lp}$; b-pour des IgG ou leurs fragments $\mathrm{F}\left(\mathrm{ab}^{\prime}\right) 2,5 \mathrm{mg} / 1 \mathrm{U}-\mathrm{Lp}$.

\section{Résultats}

Parasitémie, complexes Ig-Lp et anti-lipoprotéines.

Au cours des expériences menées lors de ce travail, l'évolution de la parasitémie et des complexes Ig-Lp confirme nos précédents résultats (Demonchy et coll., 1982; Camus et coll., 1985). En particulier, le maximum de la parasitémie exprimé en pourcentage des globules rouges infectés, est atteint 7 jours après le début de l'infection et celle-ci se résout spontanément après le $14^{\mathrm{e}}$ jour. Les complexes IgG-Lp ne sont décelés dans le sérum qu'à partir du $9^{e}$ jour, atteignent un taux maximum. au $13^{\mathrm{e}}$ jour et ne sont plus détectés au $21^{\mathrm{e}}$ jour. Les anticorps anti-lipoprotéines ne sont observés qu'au $11^{\mathrm{e}}$ jour par la technique de précipitation utilisée mais persistent après la disparition des complexes IgG-Lp du sérum ( $f g$. . 1).

Rôle immunorégulateur des lipoprotéines isolées de sérums de souris infectées par Plasmodium chabaudi.

Il peut être déduit des résultats exposés ci-dessus que les complexes Ig-Lp apparaissent au cours de la deuxième semaine d'infection, sont éliminés du courant 


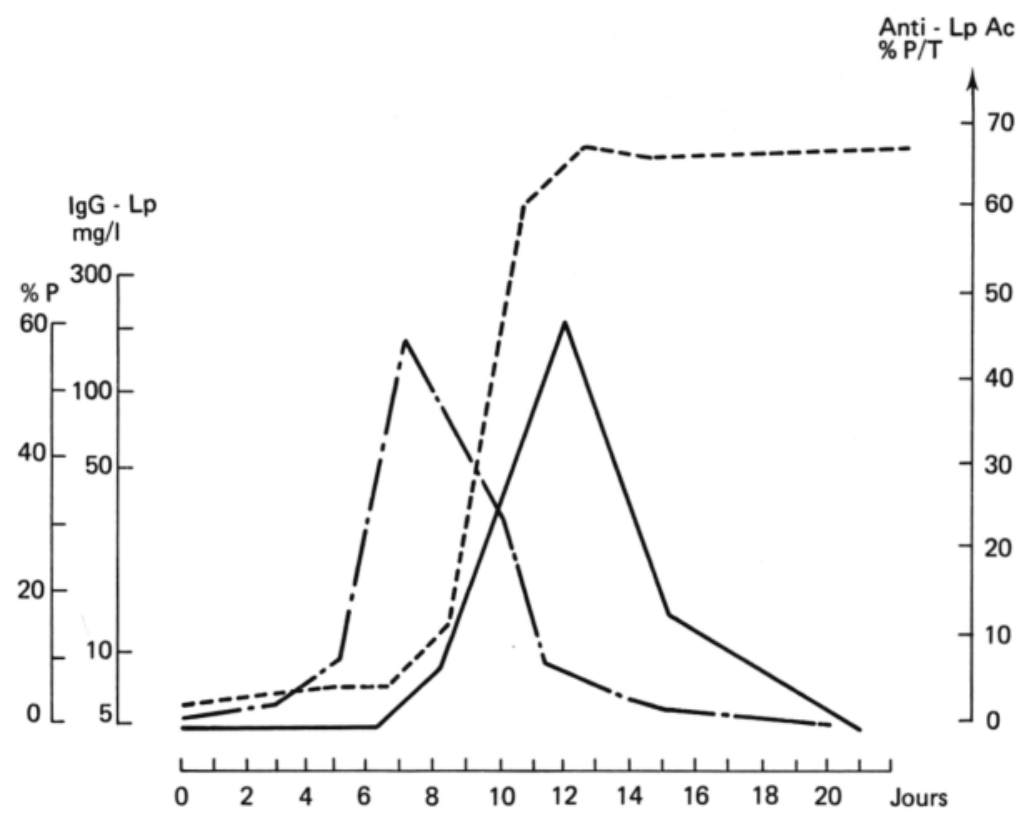

FIG. I. - Courbes d'évolution de la parasitémie (-..-) exprimée en pourcentage du nombre de globules rouges infectés $(\% \mathrm{P})$, des complexes IgG-Lp $(--)$ et des taux d'anticorps antiLp (--) exprimés en pourcentage de la radioactivité précipitée par rapport à la radioactivité totale introduite $(\% \mathrm{P} / \mathrm{T})$, chez des souris Swiss infectées par $P$. chabaudi.

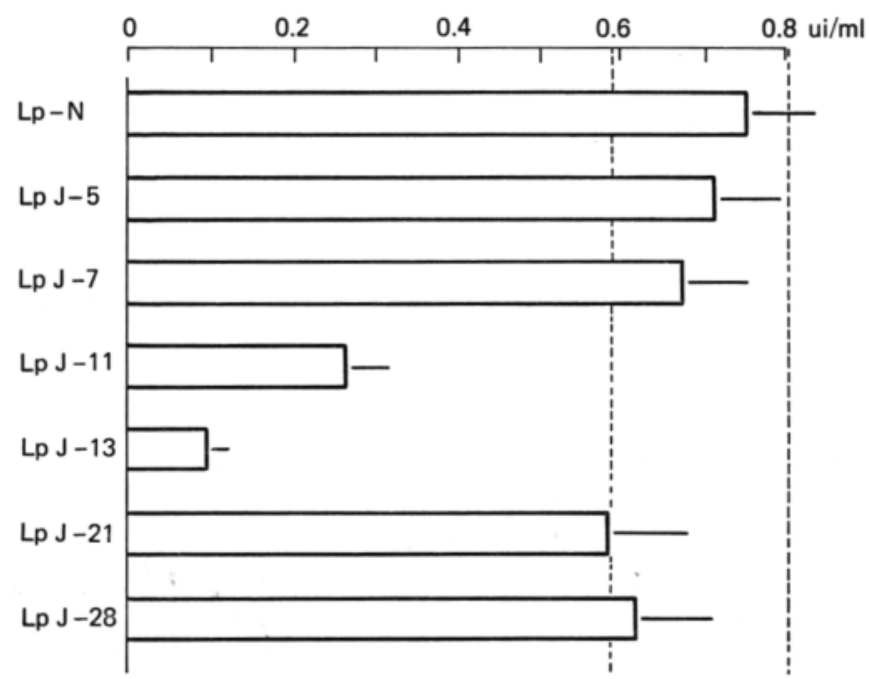

FIG. 2. - Des lipoprotéines isolées de sérums de souris infectées par $P$. chabaudi (voir le texte pour l'explication du code utilisé) sont introduites dans des cultures de cellules sécrétrices d'anticorps antitétaniques. Les anticorps sont dosés dans les surnageants des milieux de culture et leurs taux exprimés en unités internationales (ui/ml.). Les lignes en pointillé représentent les valeurs extrêmes observées dans les cultures réalisées à partir de cellules provenant de souris de contrôle immunisées vis-à-vis de l'anatoxine tétanique. Chaque moyenne et déviation standard a été établie à partir de 7 à I I déterminations. 
circulatoire au cours de la troisième semaine et que lors de la quatrième semaine les anticorps anti-lipoprotéines sont libres ou faiblement complexés. Compte tenu de ces observations, des lipoprotéines collectées par chromatographie d'affinité à différentes périodes d'infection ont été introduites dans des cultures de cellules sécrétant des anticorps antitétaniques.

Les résultats regroupés dans la figure 2, révèlent que les lipoprotéines isolées à partir des sérums J-11 ou J-13 inhibent la production d'anticorps antitétaniques in vitro. A l'inverse, les lipoprotéines sériques isolées à J-5, J-7, J-21, J-28 ou provenant de souris normales n'ont pas révélé d'activité inhibitrice sur les cellules productrices d'anticorps antitétaniques.

Des lipoprotéines isolées de sérums de souris infectées depuis 7 jours ( $\mathrm{Lp}$-J7) ont donc été incubées avec du sérum de J-20 contenant des anticorps anti-lipoprotéines Cette fraction, introduite dans des cultures de cellules productrices d'anticorps, induit une inhibition de la synthèse d'anticorps alors que les complexes (Lp-J7)$(\operatorname{ser} \mathrm{N})$ ou $\left(\mathrm{Lp}_{\mathrm{p}} \mathrm{N}\right)$-(ser J-20) n'exercent pas une telle activité (fig. 3).

Des fractions obtenues par incubation de Lp-J7 avec, soit des sérums de souris infectées depuis 20 jours, soit les IgG purifiées de ces sérums ou encore les fragments $\mathrm{F}\left(\mathrm{ab}^{\prime}\right) 2$ des IgG ont également été testées sur des cellules productrices d'anticorps antitétaniques. Les complexes formés par incubation de sérums de J-20 ou les IgG extraites de ces sérums avec des $\mathrm{Lp}$-J7 inhibent les cellules productrices d'anticorps. A l'inverse, les complexes (Lp J-7)-(fragments $\mathrm{F}\left(\mathrm{ab}^{\prime}\right) 2$ d'IgG de J-20) n'ont pas révélé d'effet inhibiteur ( $f g .3$ ). Cette expérience a donc été complétée par l'incubation des cellules sécrétrices d'anticorps avec des fragments $F_{c}$ d'IgG à raison de $1 \mathrm{mg}$ pour $10^{6}$ cellules spléniques. Après 30 minutes de contact à $37^{\circ} \mathrm{C}$ les cellules sont lavées et mises en culture pour tester les différentes fractions lipoprotéiniques. Dans ces conditions, l'activité inhibitrice des Lp J-13 et des complexes (Lp J-7)(IgG de sérum J-20) est annulée.

\section{Discussion}

La particularité de l'évolution des complexes IgG-Lp sériques et des anticorps anti-lipoprotéines au cours de l'infection de la souris Swiss par Plasmodium chabaudi (Camus et coll., 1985) a permis d'étudier le rôle immunorégulateur des lipoprotéines produites en cours d'infection sur des cellules productrices d'anticorps antitétaniques.

Les lipoprotéines recueillies à J-11 ou J-13 exercent un rôle inhibiteur sur les cellules productrices d'anticorps, mais sachant qu'à cette date les lipoprotéines sont complexées à des Ig, il était intéressant de vérifier si l'activité immunorégulatrice nécessitait impérativement des lipoprotéines sous une forme complexée. A cet effet, deux sources de réactifs ont été utilisées :

1. Des Lp-J7 connues pour être des néo-lipoprotéines probablement de structure différente des lipoprotéines de souris non infectées (Camus et coll., 1985), mais non complexées à des IgG à cette période de l'infection ( $f \mathrm{~g}$. 1).

2. Des sérums de souris infectées par $P$. chabaudi depuis 20 jours, contenant des 

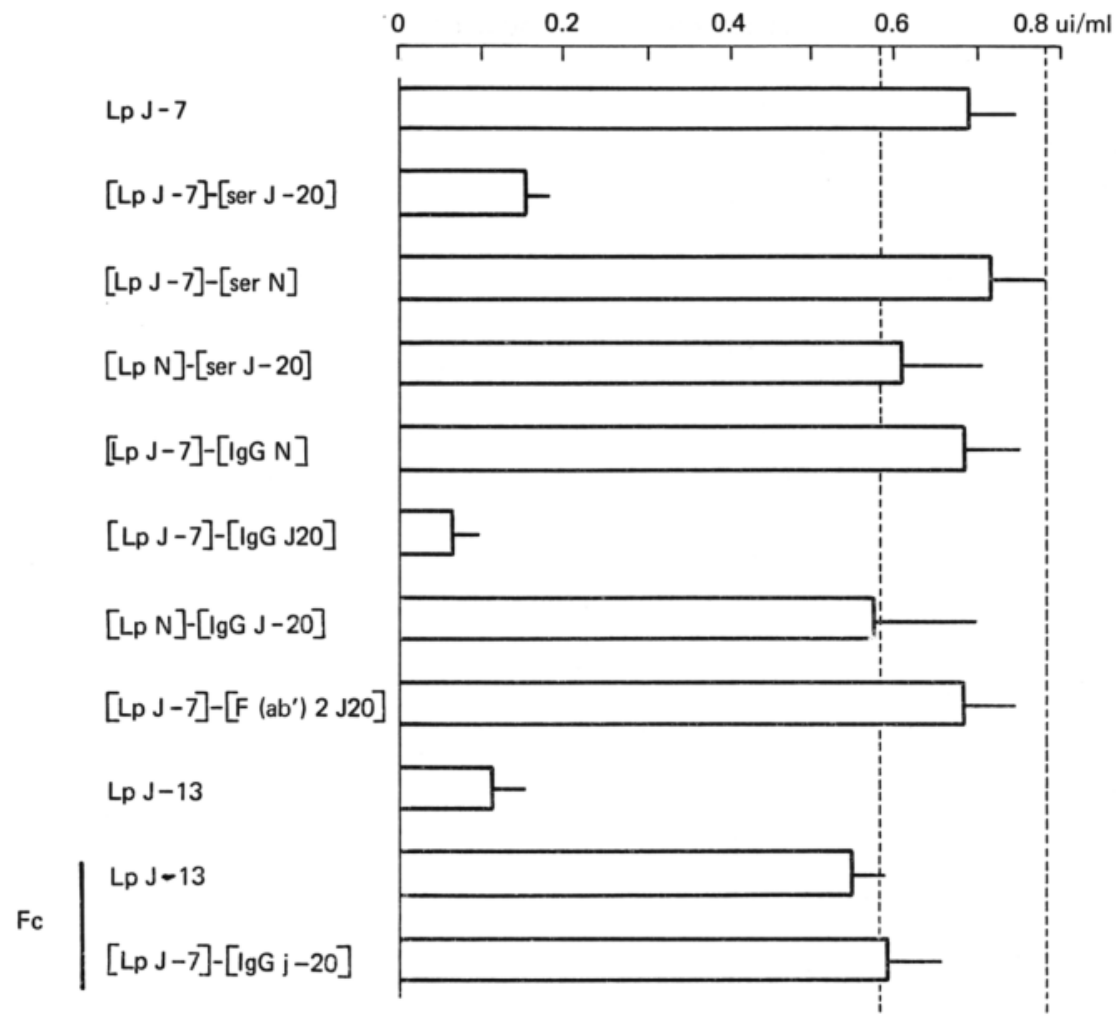

FIG. 3. - Des lipoprotéines ( $\mathrm{Lp} \mathrm{N}, \mathrm{Lp} \mathrm{J-7,} \mathrm{Lp} \mathrm{J-I3)} \mathrm{ou} \mathrm{des} \mathrm{complexes} \mathrm{formés} \mathrm{à} \mathrm{partir} \mathrm{des}$ lipoprotéines (voir le texte pour l'explication du code utilisé) sont introduits dans des cultures de cellules sécrétrices d'anticorps antitétaniques. Lors de certains protocoles les cellules ont été pré-incubées avec des fragments Fc d'IgG (Fc-). Les anticorps sont recherchés dans les surnageants des milieux de culture et leurs taux exprimés en unités internationales (ui/ml.). Les lignes en pointillé représentent les valeurs extrêmes observées dans les cultures réalisées à partir de cellules provenant de souris de contrôle immunisées vis-à-vis de l'anatoxine tétanique. Chaque moyenne et déviation standard a été établie à partir de 6 à 8 déterminations.

anticorps anti-lipoprotéines en grande partie libres puisqu'à cette période de l'infection les complexes IgG-Lp ne sont plus détectés dans le sérum (fig. 1). L'effet inhibiteur obtenu sur les cellules productrices d'anticorps antitétaniques en utilisant les complexes formés par incubation in vitro de Lp J-7 avec du sérum J-20, est donc, un argument en faveur de la nécessité d'un complexe Ig-Lp pour qu'un effet inhibiteur puisse s'exercer. De plus, malgré la présence de complexes entre lipoprotéines et immunoglobulines de type $\mathrm{M}$ en cours d'infection par $P$. chabaudi (Demonchy et coll., 1982), les résultats de la figure 3 permettent de conclure à l'effet inhibiteur des complexes formés avec des IgG. Enfin, l'absence d'effet inhibiteur des Lp J-7 incubées avec des IgG de souris non infectées suggère que les complexes inhibiteurs sont formés avec des anticorps anti-lipoprotéines. 
Les résultats rapportés au cours de notre étude complètent les informations précédemment acquises concernant le rôle immunorégulateur des lipoprotéines contenues dans des sérums normaux. L(ow) D(ensity) L(ipoproteins) et V(ery) L(ow) $\mathrm{D}$ (ensity) L(ipoproteins) exercent un rôle immunodépresseur sur les cellules lymphocytaires stimulées par des mitogènes mais aussi sur les cellules sécrétrices d'anticorps (Chisari, 1977 ; Morse et coll., 1977 ; Curtiss et coll., 1980 ; Hsu et coll., 1981). Les lipoprotéines apparaissent donc comme des facteurs immunorégulateurs naturels. Dans le cas de l'infection par $P$. chabaudi, il peut être envisagé que l'effet inhibiteur obtenu avec les complexes IgG-Lp correspond à l'amplification d'un phénomène physiologique. Cette hypothèse peut paraître plausible car il existe des liaisons naturelles entre Ig et lipoprotéines (Hartmann et coll., 1968) ; cependant, dans les conditions de notre expérience, les lipoprotéines isolées de sérums de souris non infectées $(\mathrm{Lp} \mathrm{N}$ ) n'ont jamais révélé d'activité inhibitrice sur les cellules productrices d'anticorps antitétaniques. Il peut donc être suggéré, que l'effet immunorégulateur des lipoprotéines sur les cellules productrices d'anticorps, au cours de l'infection par $P$. chabaudi se surajoute à l'effet immunorégulateur naturel des lipoprotéines mais selon une présentation qui soit celle d'un immuncomplexe. A ce titre, il a été observé dans les infections par $P$. chabaudi une association entre la présence d'immuncomplexes et l'altération des fonctions lymphocytaires (Cox et coll., 1983).

Le but du présent travail n'était pas de déterminer le mécanisme d'action des complexes IgG-Lp au niveau de la membrane des cellules productrices d'anticorps. Cependant, les informations concernant les rapports membrane cellulaire et lipoprotéines sont suffisamment fournies pour laisser supposer que la partie lipoprotéine du complexe IgG-Lp se fixe sur la membrane lymphocytaire au niveau d'un site récepteur spécifique (Curtiss et Edgington, 1978; Yi et coll., 1981). Cette hypothèse implique cependant d'admettre que les modifications touchant les lipoprotéines (Camus et coll., 1985), n'altèrent pas significativement la fixation des lipoprotéines à leur récepteur membranaire. L'absence d'effet inhibiteur lorsque sont utilisés les fragments $\mathrm{F}\left(\mathrm{ab} \mathrm{b}^{\prime}\right) 2$ des immunoglobulines ou lorsque les cellules spléniques sont pré-incubées avec des fragments Fc d'IgG, souligne par ailleurs le rôle fondamental de la portion $F_{c}$ des IgG intéressées dans les complexes IgG-Lp.

En définitive, il peut être avancé que les lipoprotéines produites au cours de l'infection de la souris Swiss par $P$. chabaudi, sont capables d'exercer un rôle immunodépresseur sur des cellules productrices d'anticorps. La nécessité d'un complexe IgG-Lp pour que puisse s'exprimer cet effet inhibiteur apparaît de plus comme un mécanisme original de l'activité immunorégulatrice des lipoprotéines, indépendant du rôle immunomodulateur des lipoprotéines sériques normales. Les effets des IgG-Lp observés in vitro dans le présent travail pourraient par ailleurs rendre compte de l'altération des réponses anticorps observées chez les souris infectées par $P$. chabaudi (Goumard et coll., 1982).

Remerciements. Nous tenons à remercier M. Jean-Louis Gentilini pour son aide lors des dosages des immunoglobulines par immunonéphélométrie, Madame Annick Wattez et Mademoiselle Catherine Ansel pour leur compétente collaboration technique. 


\section{BIBLIOGRAPHIE}

Camus D., Goumard P., Vernes A. : Lipoprotéines et malaria : I. Immunogénicité des lipoprotéines sériques chez la souris infectée par Plasmodium chabaudi. Ann. Parasitol. Hum. Comp., I985, 60, 523-532.

Capron A., Camus D., Dessaint J. P., Le Boubennec-Fisher E. : Impairment of immune response in parasitic infections. Ann. Immunol. (Inst. Pasteur), I977, I28 C, 54I-553.

CHISARI F. V. : Immunoregulatory properties of very low density lipoproteins in human plasma. J. Immunol., I977, IIg, $2129-2136$.

Cox H. W., HAyes M. M., Saleh S. M. : Immune complexes and immunoconglutinin interactions associated with altered lymphocytes activity in Plasmodium chabaudi infections. J. Parasitol., I983, 69, 809-813.

CuRtiss L. K., EDGington T. S. : Identification of a surface lymphocyte receptor for low density lipoprotein inhibitor, an immunoregulatory species of normal human serum low density lipoprotein. J. Clin. Invest., I978, 6I, I 298-1 308.

Curtiss L. K., De Heer D. H., EDGington T. S. : Influence of the immunoregulatory serum lipoprotein LDL-In on the in vivo proliferation and differentiation of antigen-binding and antibody secreting lymphocytes during a primary immune response. Cell. Immunol., I980, 49, I-I I.

Demonchy P., Maurois P., Camus D. : Complexes immunoglobulines-lipoprotéines dans l'infection par Plasmodium chabaudi. Immunol. Letters, I982, S, IIIIII 5.

Mc Gilvery R. W., Goldstein G. W. : In : Biochemistry. A functional approach. Sanders Company $W . B$., Philadelphia, I983, $3^{\mathrm{e}}$ ed., 560.

Goumard P., Vu DaC N., Maurois P., Camus D. : Influence of malaria on a pre-existing antibody response to heterologous antigens. Ann. Immunol. (Inst. Pasteur), I982, I33 D, 3I 3-326.

Goumard P., Vu DAC N., CAMus D. : Production d'anticorps antitétaniques in vitro. Immunol. Letters, soumis pour publication.

Hartmann L., Filitti-Wurmser S., Ollier M. P., Laudat P. : Lipides, Lipoprotéines, immunoglobulines IgM. Ann. Biol. Clin., I968, 26, 88I-889.

Hsu K.-H. L., Ghanta V. K., Hiramoto R. N. : Immunosuppressive effect of mouse serum lipoproteins. I. In vitro studies. J. Immunol., I981, I26, I909-1913.

Hudson L., Hay F. C. : In Practical immunology. Blackwell Scientific Publications, Oxford, I980, $2^{\mathrm{e}}$ ed., I92-I 99 .

Morse J. H., Witte L. D., Goodman W. S. : Inhibition of lymphocyte proliferation stimulated by lectins and allogenic cells by normal plasma lipoproteins. $J$. Exp. Med., I977, I46, I 791I 803.

PAN Y. T., KRUSki A. W., Elbein A. D. : Binding of 3 H-heparin to human plasma low density lipoproteins. Arch. Biochem. Biophys., 1978, I89, 231-236.

Stanworth D. R., TuRner M. W. : In Handbook of experimental immunology. Blackrell Scientific Publications, Oxford, 1978, $3^{\mathrm{e}}$ ed., 6, r-6, Io2.

Y I P. I., BECK G., ZUCKER S. : Rat plasma lipoprotein inhibitors of lymphocyte proliferation : specific membrane receptor for very low density lipoproteins. Int. Arch. Allergy appl. Immunol., 1981, 65, 8-14. 